\title{
Heat and Mass Transfer for MHD Viscoelastic Fluid Flow over a Vertical Stretching Sheet with Considering Soret and Dufour Effects
}

\author{
Mohammad Mehdi Rashidi, ${ }^{1,2,3}$ Mohamed Ali, ${ }^{4}$ Behnam Rostami, ${ }^{3}$ \\ Peyman Rostami, ${ }^{5}$ and Gong-Nan Xie ${ }^{6}$ \\ ${ }^{1}$ Shanghai Automotive Wind Tunnel Center, Tongji University, 4800 Caoan Road, Jiading, Shanghai 201804, China \\ ${ }^{2}$ ENN-Tongji Clean Energy Institute of Advanced Studies, Shanghai, China \\ ${ }^{3}$ Mechanical Engineering Department, Engineering Faculty, Bu-Ali Sina University, Hamedan, Iran \\ ${ }^{4}$ Mechanical Engineering Department, College of Engineering, King Saud University, P.O. Box 800, Riyadh 11421, Saudi Arabia \\ ${ }^{5}$ Department of Mechanical Engineering, Isfahan University of Technology, Isfahan 84156 83111, Iran \\ ${ }^{6}$ School of Mechanical Engineering, Northwestern Polytechnical University, Xi'an, Shaanxi, China \\ Correspondence should be addressed to Mohamed Ali; mali@ksu.edu.sa
}

Received 22 March 2014; Revised 17 June 2014; Accepted 6 September 2014

Academic Editor: Haochun Zhang

Copyright ( 2015 Mohammad Mehdi Rashidi et al. This is an open access article distributed under the Creative Commons Attribution License, which permits unrestricted use, distribution, and reproduction in any medium, provided the original work is properly cited.

\begin{abstract}
The homotopy analysis method (HAM) with two auxiliary parameters is employed to examine heat and mass transfer in a steady two-dimensional magneto hydrodynamic viscoelastic fluid flow over a stretching vertical surface by considering Soret and Dufour effects. The two-dimensional boundary-layer governing partial differential equations are derived by considering the Boussinesq approximation. The highly nonlinear ordinary differential forms of momentum, energy, and concentration equations are obtained by similarity transformation. These equations are solved analytically in the presence of buoyancy force. The effects of different involved parameters such as magnetic field parameter, Prandtl number, buoyancy parameter, Soret number, Dufour number, and Lewis number on velocity, temperature, and concentration profiles are plotted and discussed. The effect of the second auxiliary parameter is also illustrated. Results show that the effect of increasing Soret number or decreasing Dufour number tends to decrease the velocity and temperature profiles (increase in $\mathrm{Sr}$ cools the fluid and reduces the temperature) while enhancing the concentration distribution.
\end{abstract}

\section{Introduction}

The analysis of the flow field in a boundary-layer near a stretching sheet is an important part in fluid dynamics and heat transfer. This type of flow occurs in a number of engineering processes such as extrusion of plastic sheets, polymer processing, and metallurgy $[1,2]$.

Some researchers neglect the Dufour and Soret effects on heat and mass transfer according to Fourier's and Fick's laws [3]; however, when density differences exist in the flow regime, these effects are important and cannot be neglected [4]. Afify [5] has shown that when heat and mass transfer occurred in a moving fluid, the energy flux can be generated by a composition gradient, namely, the Dufour or diffusionthermo effect, and the mass fluxes developed by the temperature gradients are called the Soret or thermal-diffusion effect. In their numerical study they have used the Soret and Dufour effects of a steady flow due to a rotating disk in the presence of viscous dissipation and ohmic heating. Heat and mass transfer with hydrodynamic slip over a moving plate in porous media was investigated by Hamad et al. [6] via Runge-Kutta-Fehlberg fourth-fifth order method. The heat transfer of mixed convection of vertically moving surface in an ambient stagnant fluid was reported by Ali and Al-Yousef $[7,8]$ and the effect of variable viscosity of mixed convection was studied by Ali [9]. 
Das et al. [10] considered the effect of heat and mass transfer on a free convective flow of an incompressible electrically conducting fluid past a vertical porous plate. Chen [11] employed finite difference method in order to study the heat and mass transfer in MHD free convective flow with ohmic heating and viscous dissipation. Noor et al. [12] examined the MHD flow over an inclined surface with heat source/sink effects by shooting method. Abreu et al. [13] solved the boundary-layer flow with Dufour and Soret effects in both forced and natural convection. The effects of thermal radiation and first order chemical reaction on unsteady MHD convective flow past a semiinfinite vertical plate under oscillatory suction and heat source in slip-flow regime were taken into account by Pal and Talukdar [14]. Gbadeyan et al. [15] studied heat and mass transfer of a mixed convection boundary-layer flow considering porous medium over a stretching vertical surface. A vertical plate in a non-Darcy porous medium was selected to investigate the thermodiffusion and diffusion-thermo effects numerically using the Keller-box method by Prasad et al. [16]. Pal and Mondal [1719] analyzed the effects of thermal diffusion and diffusion thermo on steady and unsteady MHD non-Darcy flow over a stretching sheet in a porous medium considering thermal radiation, nonuniform heat source/sink, variable viscosity, viscous dissipation, and first order chemical reaction using Runge-Kutta-Fehlberg integration method. Mansour et al. [20] analyzed the effects of chemical reaction and thermal stratification over a vertical stretching surface in a porous medium by Runge-Kutta scheme with considering Soret and Dufour numbers. Bég et al. [21] used Keller-box implicit method to analyze the heat and mass transfer micropolar fluid flow from an isothermal sphere with Soret and Dufour effects. Furthermore, Alam et al. [22], Tai and Char [23], Mahdy [24, 25], Pal and Sewli [26], and Tsai and Huang [27] have studied the effect of Soret and Dufour effects in their analyses for different aspects of heat and mass transfer flows.

One of the most effective and reliable methods in order to solve the high nonlinear problems is the homotopy analysis method. Homotopy analysis method (HAM) was firstly employed by Liao to offer a general analytic method for nonlinear problems [28, 29]. Rashidi et al. [30] analyzed the effect of partial slip, diffusion thermo, and thermal diffusion on MHD fluid flow in a rotating disk via HAM and discussed the effect of various slip parameters, magnetic field parameter, Schmidt number, and other important variables. Mustafa et al. [31] considered the effects of Brownian motion and thermophoresis in stagnation point flow of a nanofluid towards a stretching sheet. Rashidi and Pour [32] employed HAM for unsteady boundary-layer flow and heat transfer on a stretching sheet. Abbas et al. [33] studied the mixed convection of an incompressible Maxwell fluid flow over a vertical stretching surface by HAM. Dinarvand et al. [34] employed HAM to investigate unsteady laminar MHD flow near forward stagnation point of a rotating and translating sphere. Hayat et al. [35] illustrated the thermal-diffusion and diffusionthermo effects on two-dimensional MHD axisymmetric flow of a second grade fluid in the presence of Joule heating and first order chemical reaction. The non-linear Brinkman equation for the stagnation-point flow was studied via HAM by Ziabakhsh et al. [36]. Analytical and numerical solutions of a radial stagnation flow over a stretching cylinder have been recently reported by Weidman and Ali [37] where aligned and nonaligned flow were studied. Rashidi et al. [38, 39] employed HAM to obtain the analytical solutions over stretching and shrinking sheets in the presence of buoyancy parameter.

The objective of this paper is to study the steady twodimensional MHD viscoelastic fluid flow over a vertical stretching surface in the presence of the Soret and Dufour effects analytically via HAM. The effects of different involved parameters such as magnetic field parameter, Prandtl number, buoyancy parameter, Soret number, Dufour number, and Lewis number on the fluid velocity, temperature, and concentration distributions are plotted and discussed.

\section{Flow Analysis}

Consider a steady two-dimensional heat and mass transfer flow of an incompressible electrically conducting viscoelastic fluid over a stretching vertical surface with a variable magnetic field $B(x)=B_{0} x^{(n-1) / 2}$ normally applied to the surface. Keeping the origin fixed, two equal and opposite forces are applied along the $x$-axis. It is assumed that the stretching velocity is in the form of $u_{w}(x)=a x^{n}$, where $a$ and $n$ are constants. The induced magnetic field is neglected in comparison to the applied magnetic field and the viscous dissipation is small. The governing equations subject to Boussinesq approximation, the boundary-layer assumptions, and the above assumptions can be written as (for more details see [41])

$$
\begin{gathered}
\frac{\partial u}{\partial x}+\frac{\partial v}{\partial y}=0 \\
u \frac{\partial u}{\partial x}+v \frac{\partial u}{\partial y} \\
=v \frac{\partial^{2} u}{\partial y^{2}}+k_{0}\left(u \frac{\partial^{3} u}{\partial x \partial y^{2}}+\frac{\partial u}{\partial x} \frac{\partial^{2} u}{\partial y^{2}}+\frac{\partial u}{\partial y} \frac{\partial^{2} v}{\partial y^{2}}+v \frac{\partial^{3} u}{\partial y^{3}}\right) \\
-\frac{\sigma B^{2}(x) u}{\rho}+g\left(\beta_{T}\left(T-T_{\infty}\right)+\beta_{C}\left(C-C_{\infty}\right)\right) \\
u \frac{\partial T}{\partial x}+v \frac{\partial T}{\partial y}=\alpha \frac{\partial^{2} T}{\partial y^{2}}+\frac{D_{e} k_{T}}{c_{s} c_{P}} \frac{\partial^{2} C}{\partial y^{2}} \\
u \frac{\partial C}{\partial x}+v \frac{\partial C}{\partial y}=D_{e} \frac{\partial^{2} C}{\partial y^{2}}+\frac{D_{e} k_{T}}{T_{m}} \frac{\partial^{2} T}{\partial y^{2}}
\end{gathered}
$$

where $u$ and $v$ are velocity components in the directions of $x$ and $y$ along and normal to the surface, respectively (as shown in Figure 1). $v$ is the kinematic viscosity, $k_{0}$ is the viscoelasticity parameter, $\sigma$ is the electrical conductivity, $\rho$ is the fluid density, $g$ is the acceleration due to gravity, $\beta_{T}$ is the coefficient of thermal expansion, $\beta_{C}$ is the coefficient of thermal expansion with concentration, $\alpha$ is the thermal diffusivity, $k_{T}$ is the thermal diffusion ratio, $c_{s}$ is the concentration susceptibility, $c_{P}$ is the specific heat at constant pressure, $D_{e}$ is the coefficient of mass diffusivity, $T$ is the fluid 


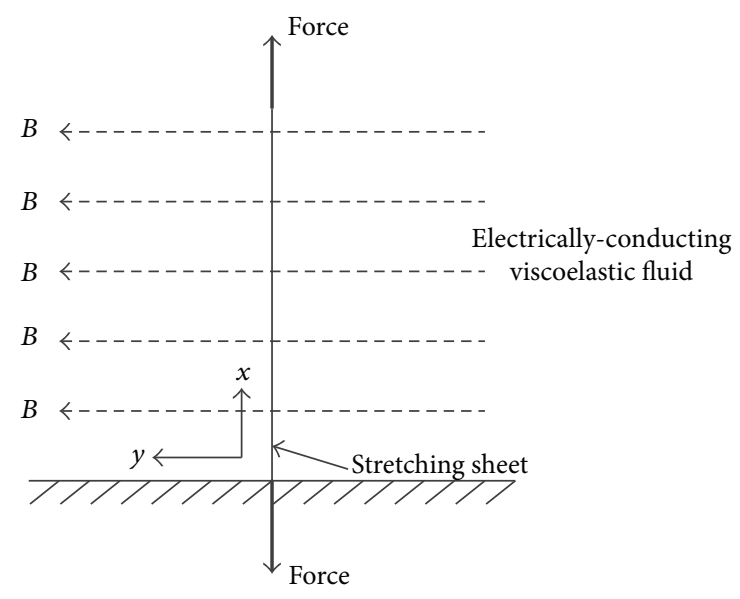

FIGURE 1: The schematic diagram of the stretching sheet problem.

temperature, $C$ is the fluid concentration, and $T_{m}$ is the mean fluid temperature.

The corresponding boundary conditions are as follows:

$$
\begin{aligned}
& u=u_{w}(x), \\
& v=v_{w}, \\
& T=T_{w}(x), \\
& C=C_{w}(x) \\
& \text { at } y=0, \\
& u \longrightarrow 0, \\
& \partial u \\
& \frac{\partial y}{\longrightarrow} 0, \\
& T \longrightarrow T_{\infty}, \\
& C \longrightarrow C_{\infty}
\end{aligned}
$$

We assume that $T_{w}(x)=T_{\infty}+b x$ and $C_{w}(x)=C_{\infty}+c x$, where $b$ and $c$ are constants. Introducing stream function $\psi$, and similarity variable $\eta$, [42] the continuity equation is satisfied and the momentum, energy, and concentration equations are transformed into ordinary differential equations as follows:

$$
\begin{gathered}
\eta=\sqrt{\frac{u_{w}}{v x}} y, \\
\psi=\sqrt{u_{w} v x} f(\eta), \\
\theta(\eta)=\frac{T-T_{\infty}}{T_{w}-T_{\infty}}, \\
\varphi(\eta)=\frac{C-C_{\infty}}{C_{w}-C_{\infty}},
\end{gathered}
$$

$$
\begin{aligned}
& n f^{\prime 2}-\frac{n+1}{2} f f^{\prime \prime}-f^{\prime \prime \prime} \\
& -k_{1}\left\{(3 n-1) f^{\prime} f^{\prime \prime \prime}-\frac{(3 n-1)}{2} f^{\prime \prime 2}-\frac{(n+1)}{2} f f^{(4)}\right\} \\
& +\operatorname{Mn} f^{\prime}-\lambda(\theta+N \varphi)=0, \\
& \theta^{\prime \prime}+\operatorname{Pr}\left(\frac{n+1}{2} f \theta^{\prime}-f^{\prime} \theta+\operatorname{Du} \varphi^{\prime \prime}\right)=0, \\
& \varphi^{\prime \prime}+\operatorname{Le}\left\{\operatorname{Pr}\left(\frac{n+1}{2} f \varphi^{\prime}-f^{\prime} \varphi\right)+\operatorname{Sr} \theta^{\prime \prime}\right\}=0,
\end{aligned}
$$

where superscript $I$ denotes the derivative with respect to $\eta$, $k_{1}=k_{0} a x^{n-1} / v$ is the viscoelasticity parameter (when $n=1$ the viscoelastic parameter takes the form of $k_{1}=k_{0} a / v$ similar to the viscoelastic parameter obtained by Hayat et al. [41]), $\mathrm{Mn}=\sigma B_{0}^{2} / a \rho$ is the magnetic field parameter, $\lambda=g \beta_{T}\left(T_{w}-T_{\infty}\right) x / a^{2} x^{(2 n-1)} x=\mathrm{Gr}_{x} / \operatorname{Re}_{x}^{2}$ is the buoyancy parameter, where $\mathrm{Gr}_{x}=g \beta_{T}\left(T_{w}-T_{\infty}\right) x^{3} / v^{2}$ is the Grashof number, $\operatorname{Re}_{x}=u_{w} x / v$ is the Reynolds number, $N=$ $\beta_{C}\left(C_{w}-C_{\infty}\right) / \beta_{T}\left(T_{w}-T_{\infty}\right)$ is the constant dimensionless concentration buoyancy parameter, $\operatorname{Pr}=v / \alpha$ is the Prandtl number, Le $=\alpha / D_{e}$ is the Lewis number, $\mathrm{Sr}=D_{e} k_{T}\left(T_{w}-\right.$ $\left.T_{\infty}\right) / T_{m} \alpha\left(C_{w}-C_{\infty}\right)$ is the Soret number, and $\mathrm{Du}=$ $D_{e} k_{T}\left(C_{w}-C_{\infty}\right) / c_{s} c_{P}\left(T_{w}-T_{\infty}\right) v$ is the Dufour number. The corresponding boundary conditions are as follows:

$$
\begin{aligned}
& f(\eta)=f_{w}, \\
& f^{\prime}(\eta)=1, \\
& \theta(\eta)=1, \\
& \varphi(\eta)=1 \\
& \text { at } \eta=0, \\
& f^{\prime}(\eta)=0, \\
& f^{\prime \prime}(\eta)=0, \\
& \theta(\eta)=0, \\
& \varphi(\eta)=0 \\
& \text { as } \eta \longrightarrow \infty,
\end{aligned}
$$

where $v_{w}=-f_{w} \sqrt{a v}((n+1) / 2) x^{(n-1) / 2}$ is the suction/injection parameter ( $f_{w}>0$ for suction and $f_{w}<0$ for injection). In this paper the suction parameter has been considered because the primary assumption in boundary-layer definition says that the boundary-layer thickness is supposed to be very thin and we are not allowed to increase it, so we do not present the injection parameters that may lead to enlarging the boundary-layer thickness and contravening the boundarylayer assumption presented by Prandtl in 1904. 


\section{HAM Solution}

We choose the initial approximations to satisfy the boundary conditions. The appropriate initial approximations are as follows:

$$
\begin{aligned}
& f_{0}(\eta)=f_{w}+\frac{\left(1-e^{-\gamma \eta}\right)}{\gamma}, \\
& \theta_{0}(\eta)=e^{-\gamma \eta}, \\
& \varphi_{0}(\eta)=e^{-\gamma \eta},
\end{aligned}
$$

where $\gamma$ is the second auxiliary parameter. The linear operators $\mathscr{L}_{f}(f), \mathscr{L}_{\theta}(\theta)$ and $\mathscr{L}_{\varphi}(\varphi)$ are

$$
\begin{aligned}
& \mathscr{L}_{f}(f)=\frac{\partial^{4} f}{\partial \eta^{4}}+\gamma \frac{\partial^{3} f}{\partial \eta^{3}}, \\
& \mathscr{L}_{\theta}(\theta)=\frac{\partial^{2} \theta}{\partial \eta^{2}}+\gamma \frac{\partial \theta}{\partial \eta} \\
& \mathscr{L}_{\varphi}(\varphi)=\frac{\partial^{2} \varphi}{\partial \eta^{2}}+\gamma \frac{\partial \varphi}{\partial \eta}
\end{aligned}
$$

with the following properties:

$$
\begin{aligned}
\mathscr{L}_{f}\left(c_{1}+c_{2} \eta+c_{3} \eta^{2}+c_{4} e^{-\gamma \eta}\right) & =0, \\
\mathscr{L}_{\theta}\left(c_{5}+c_{6} e^{-\gamma \eta}\right) & =0, \\
\mathscr{L}_{\varphi}\left(c_{7}+c_{8} e^{-\gamma \eta}\right) & =0,
\end{aligned}
$$

where $c_{1}-c_{8}$ are arbitrary constants and the nonlinear operators are

$$
\begin{aligned}
& \mathcal{N}_{f}[\widehat{f}(\eta ; q), \widehat{\theta}(\eta ; q), \widehat{\varphi}(\eta ; q)] \\
& =n\left(\frac{\partial \widehat{f}(\eta ; q)}{\partial \eta}\right)^{2}-\frac{n+1}{2} \widehat{f}(\eta ; q) \frac{\partial^{2} \widehat{f}(\eta ; q)}{\partial \eta^{2}}-\frac{\partial^{3} \widehat{f}(\eta ; q)}{\partial \eta^{3}} \\
& -k_{1}\left\{(3 n-1) \frac{\partial \widehat{f}(\eta ; q)}{\partial \eta} \frac{\partial^{3} \widehat{f}(\eta ; q)}{\partial \eta^{3}}\right. \\
& -\frac{n+1}{2} \widehat{f}(\eta ; q) \frac{\partial^{4} \widehat{f}(\eta ; q)}{\partial \eta^{4}} \\
& \left.\quad-\frac{3 n-1}{2}\left(\frac{\partial^{2} \widehat{f}(\eta ; q)}{\partial \eta^{2}}\right)^{2}\right\} \\
& +\operatorname{Mn} \frac{\partial \widehat{f}(\eta ; q)}{\partial \eta} \\
& -\lambda(\widehat{\theta}(\eta ; q)+N \widehat{\varphi}(\eta ; q)),
\end{aligned}
$$

$$
\begin{aligned}
& \mathcal{N}_{\theta} {[\widehat{f}(\eta ; q), \widehat{\theta}(\eta ; q), \widehat{\varphi}(\eta ; q)] } \\
&= \frac{\partial^{2} \widehat{\theta}(\eta ; q)}{\partial \eta^{2}} \\
&+\operatorname{Pr}\left(\frac{n+1}{2} \widehat{f}(\eta ; q) \frac{\partial \hat{\theta}(\eta ; q)}{\partial \eta}-\frac{\partial \widehat{f}(\eta ; q)}{\partial \eta} \widehat{\theta}(\eta ; q)\right) \\
&+\operatorname{Pr} \cdot \operatorname{Du} \frac{\partial^{2} \widehat{\varphi}(\eta ; q)}{\partial \eta^{2}}, \\
& \mathcal{N}_{\varphi}[\widehat{f}(\eta ; q), \widehat{\theta}(\eta ; q), \widehat{\varphi}(\eta ; q)] \\
&=\frac{\partial^{2} \widehat{\varphi}(\eta ; q)}{\partial \eta^{2}} \\
& \quad+\operatorname{Pr} \cdot \operatorname{Le}\left(\frac{n+1}{2} \widehat{f}(\eta ; q) \frac{\partial \hat{\varphi}(\eta ; q)}{\partial \eta}-\frac{\partial \widehat{f}(\eta ; q)}{\partial \eta} \widehat{\varphi}(\eta ; q)\right) \\
&+\operatorname{Sr} \cdot \operatorname{Le} \frac{\partial^{2} \widehat{\theta}(\eta ; q)}{\partial \eta^{2}} .
\end{aligned}
$$

The auxiliary functions are introduced as

$$
\mathscr{H}_{f}(\eta)=\mathscr{H}_{\theta}(\eta)=\mathscr{H}_{\varphi}(\eta)=e^{-\gamma \eta} .
$$

The ith order deformation equations (see (10)) can be solved by the symbolic software MATHEMATICA

$$
\begin{aligned}
& \mathscr{L}_{f}\left[f_{i}(\eta)-\chi_{i} f_{i-1}(\eta)\right]=\hbar \mathscr{H}_{f}(\eta) R_{f, i}(\eta), \\
& \mathscr{L}_{\theta}\left[\theta_{i}(\eta)-\chi_{i} \theta_{i-1}(\eta)\right]=\hbar \mathscr{H}_{\theta}(\eta) R_{\theta, i}(\eta), \\
& \mathscr{L}_{\varphi}\left[\varphi_{i}(\eta)-\chi_{i} \varphi_{i-1}(\eta)\right]=\hbar \mathscr{H}_{\varphi}(\eta) R_{\varphi, i}(\eta),
\end{aligned}
$$

where $\hbar$ is the auxiliary nonzero parameter

$$
\begin{aligned}
& R_{f, i}(\eta) \\
& =\sum_{j=0}^{i-1}\left(n \frac{\partial f_{j}(\eta)}{\partial \eta} \frac{\partial f_{i-1-j}(\eta)}{\partial \eta}-\frac{n+1}{2} f_{j}(\eta) \frac{\partial^{2} f_{i-1-j}(\eta)}{\partial \eta^{2}}\right) \\
& -\frac{\partial^{3} f_{i-1}(\eta)}{\partial \eta^{3}} \\
& -\sum_{j=0}^{i-1} k_{1}\left((3 n-1) \frac{\partial f_{j}(\eta)}{\partial \eta} \frac{\partial^{3} f_{i-1-j}(\eta)}{\partial \eta^{3}}\right. \\
& \quad-\frac{n+1}{2} f_{j}(\eta) \frac{\partial^{4} f_{i-1-j}(\eta)}{\partial \eta^{4}} \\
& \left.\quad-\left(\frac{3 n-1}{2}\right) \frac{\partial^{2} f_{j}(\eta)}{\partial \eta^{2}} \frac{\partial^{2} f_{i-1-j}(\eta)}{\partial \eta^{2}}\right) \\
& +\operatorname{Mn} \frac{\partial f_{i-1}(\eta)}{\partial \eta}-\lambda\left(\theta_{i-1}(\eta)+N \varphi_{i-1}(\eta)\right),
\end{aligned}
$$




$$
\begin{aligned}
& R_{\theta, i}(\eta) \\
& =\frac{\partial^{2} \theta_{i-1}(\eta)}{\partial \eta^{2}} \\
& +\operatorname{Pr} \sum_{j=0}^{i-1}\left(\frac{n+1}{2} f_{j}(\eta) \frac{\partial \theta_{i-1-j}(\eta)}{\partial \eta}\right. \\
& \left.-\theta_{j}(\eta) \frac{\partial f_{i-1-j}(\eta)}{\partial \eta}\right) \\
& +\operatorname{Pr} \cdot \operatorname{Du} \frac{\partial^{2} \varphi_{i-1}(\eta)}{\partial \eta^{2}}, \\
& R_{\varphi, i}(\eta) \\
& =\frac{\partial^{2} \varphi_{i-1}(\eta)}{\partial \eta^{2}} \\
& +\operatorname{Pr} \cdot \operatorname{Le} \sum_{j=0}^{i-1}\left(\frac{n+1}{2} f_{j}(\eta) \frac{\partial \varphi_{i-1-j}(\eta)}{\partial \eta}\right. \\
& \left.-\varphi_{j}(\eta) \frac{\partial f_{i-1-j}(\eta)}{\partial \eta}\right) \\
& +\operatorname{Sr} \cdot \operatorname{Le} \frac{\partial^{2} \theta_{i-1}(\eta)}{\partial \eta^{2}} \\
& \chi_{i}= \begin{cases}0, & i \leq 1, \\
1, & i>1 .\end{cases}
\end{aligned}
$$

For more information about the HAM solution, see [28, 29].

In Figure $2, \hbar$-curve is figured, obtained via 20th order of HAM solution. The averaged residual errors are defined as (12) to acquire optimal values of auxiliary parameters:

$$
\begin{aligned}
& \operatorname{Res}_{f} \\
& =n\left(\frac{d f(\eta)}{d \eta}\right)^{2}-\frac{n+1}{2} f(\eta) \frac{d^{2} f(\eta)}{d \eta^{2}} \\
& -\frac{d^{3} f(\eta)}{d \eta^{3}}+\operatorname{Mn} \frac{d f(\eta)}{d \eta} \\
& -k_{1}\left\{(3 n-1) \frac{d f(\eta)}{d \eta} \frac{d^{3} f(\eta)}{d \eta^{3}}\right. \\
& -\lambda(\theta(\eta)+N \varphi(\eta)), \\
& \left.\operatorname{Res}_{\theta} \quad-\frac{n+1}{2} f(\eta) \frac{d^{4} f(\eta)}{d \eta^{4}}-\frac{3 n-1}{2}\left(\frac{d^{2} f(\eta)}{d \eta^{2}}\right)^{2}\right\} \\
& =\frac{d^{2} \theta(\eta)}{d \eta^{2}} \\
& \quad+\operatorname{Pr}\left(\left(\frac{n+1}{2}\right) f(\eta) \frac{d \theta(\eta)}{d \eta}\right. \\
& \left.\quad-\frac{d f(\eta)}{d \eta} \theta(\eta)+\operatorname{Du} \frac{d^{2} \varphi(\eta)}{d \eta^{2}}\right),
\end{aligned}
$$

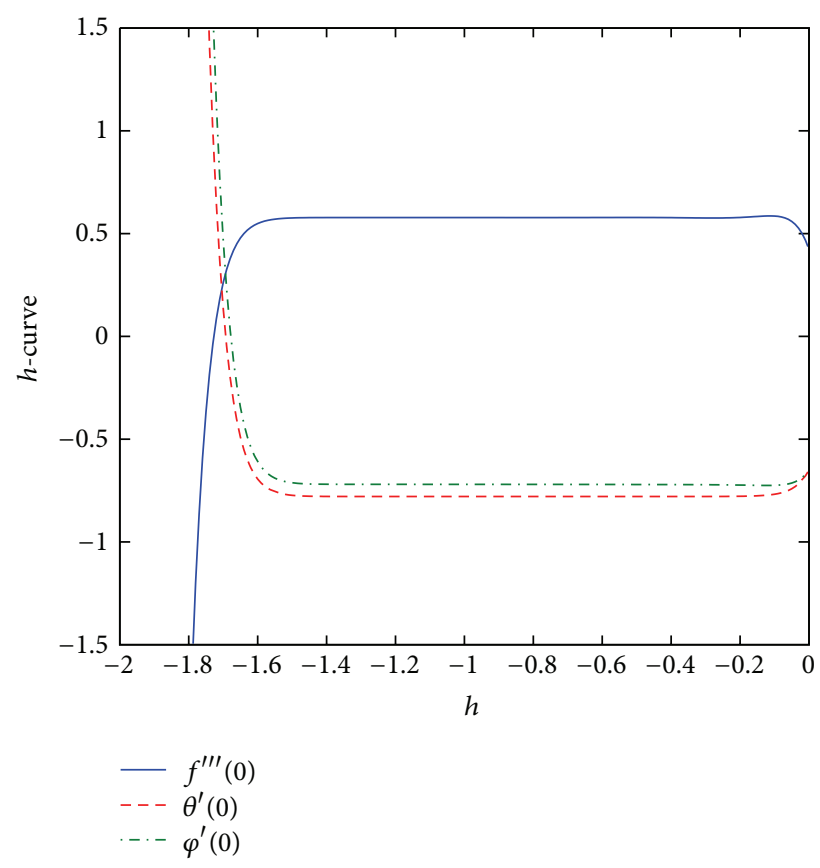

FIgURE 2: The $\hbar$-curves of $f^{\prime \prime \prime}(0), \theta^{\prime}(0)$, and $\varphi^{\prime}(0)$ obtained by the 20th order approximation of the HAM solution when $k_{1}=1, \mathrm{Mn}=$ $0.5, \lambda=0.6, \mathrm{Pr}=0.71, \mathrm{Du}=0.2, \mathrm{Sr}=0.25, n=0.5, \gamma=0.65$, $f_{w}=0.1$, and $\mathrm{Le}=1$.

$$
\begin{aligned}
& \operatorname{Res}_{\varphi} \\
& =\frac{d^{2} \varphi(\eta)}{d \eta^{2}} \\
& \quad+\operatorname{Pr} \cdot \operatorname{Le}\left(\left(\frac{n+1}{2}\right) f(\eta) \frac{d \varphi(\eta)}{d \eta}-\frac{d f(\eta)}{d \eta} \varphi(\eta)\right) \\
& \quad+\operatorname{Sr} \cdot \operatorname{Le} \frac{d^{2} \theta(\eta)}{d \eta^{2}} .
\end{aligned}
$$

To check the accuracy of the method, the residual errors of (12) are illustrated in Figures 3 and 4. The residual errors are reduced when we use the second auxiliary parameter and this justifies why we use the second auxiliary parameter. In Figure 3 , the effect of considering $\gamma=0.65$ is to decrease the order of residual errors than at $\gamma=1$ (without the second auxiliary parameter) in Figure 4 which improves the accuracy of the HAM method. The velocity profiles presented in Figure 5 show an excellent agreement between our results and [40].

\section{Results and Discussion}

In this paper the MHD two-dimensional steady heat and mass transfer flow of an incompressible viscoelastic fluid over a stretching vertical surface with considering the effects of Soret and Dufour numbers is investigated. Applying numerical values to the problem parameters, we can discuss their 


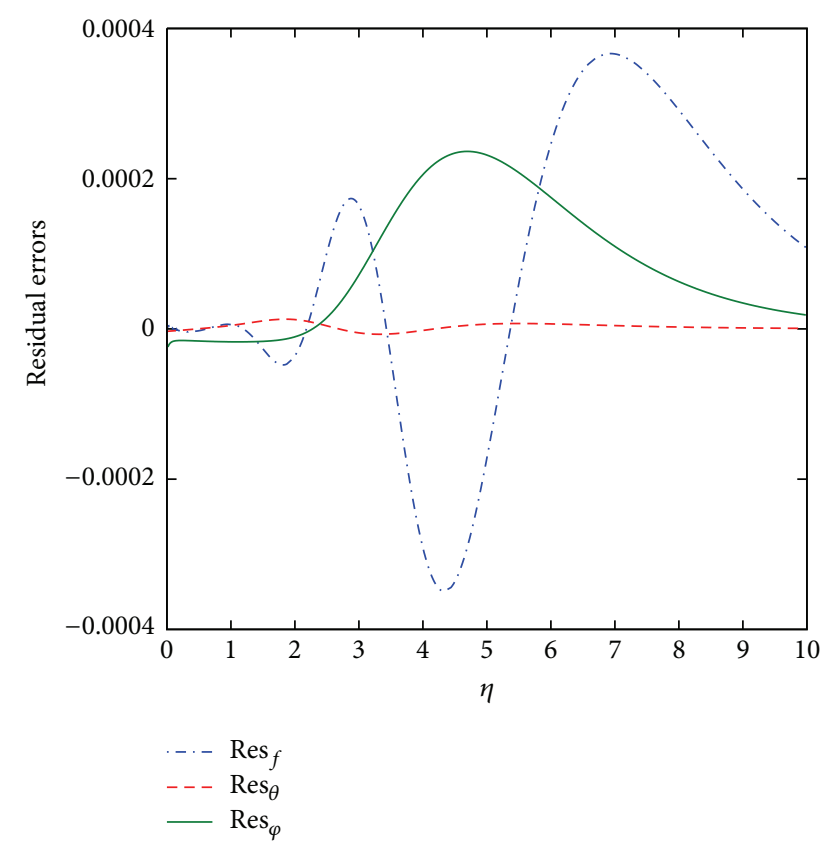

Figure 3: The residual errors when $k_{1}=1, \mathrm{Mn}=0.5, \lambda=0.6$, $\operatorname{Pr}=0.71, \mathrm{Du}=0.2, \mathrm{Sr}=0.25, n=0.5, \mathrm{Le}=1, f_{w}=0.1$, and $\gamma=0.65$.

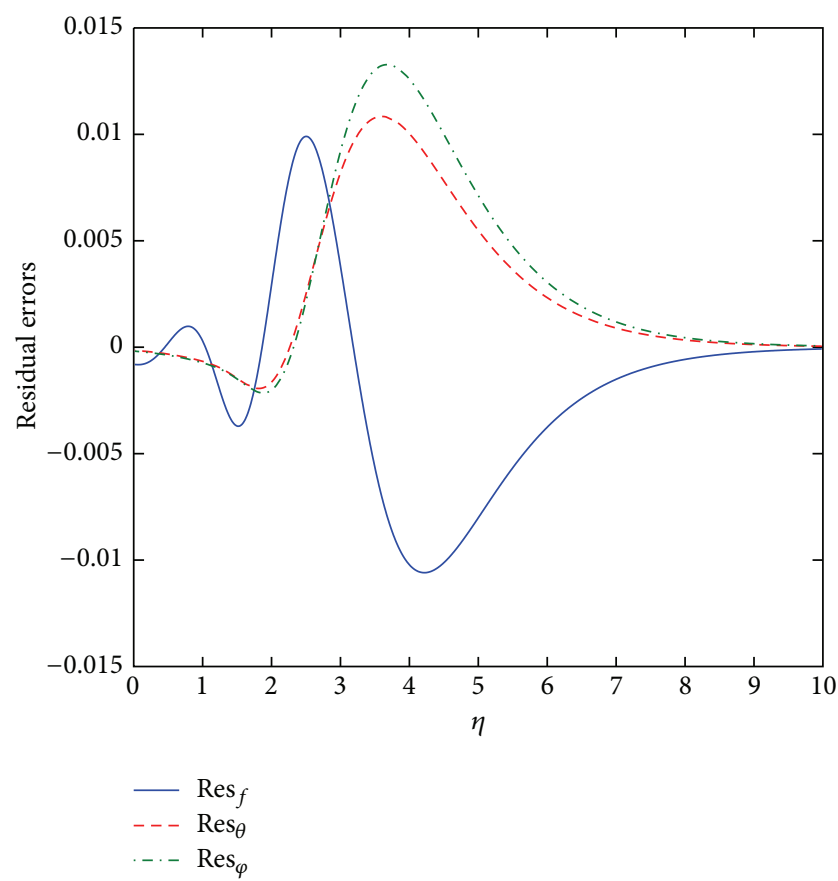

FIGURE 4: The residual errors when $k_{1}=1, \mathrm{Mn}=0.5, \lambda=0.6$, $\operatorname{Pr}=0.71, \mathrm{Du}=0.2, \mathrm{Sr}=0.25, n=0.5, \mathrm{Le}=1, f_{w}=0.1$, and $\gamma=1$.

effects on the velocity $f^{\prime}$, temperature $\theta$, and concentration $\varphi$ distributions. Graphical illustration of the results is very useful and practical to discuss the effect of different parameters. In this analysis, it has been considered that $N=-0.5$ [41]. Negative $N$ (thermal and concentration buoyancy forces oppose each other) induces a slight increase in both fluid

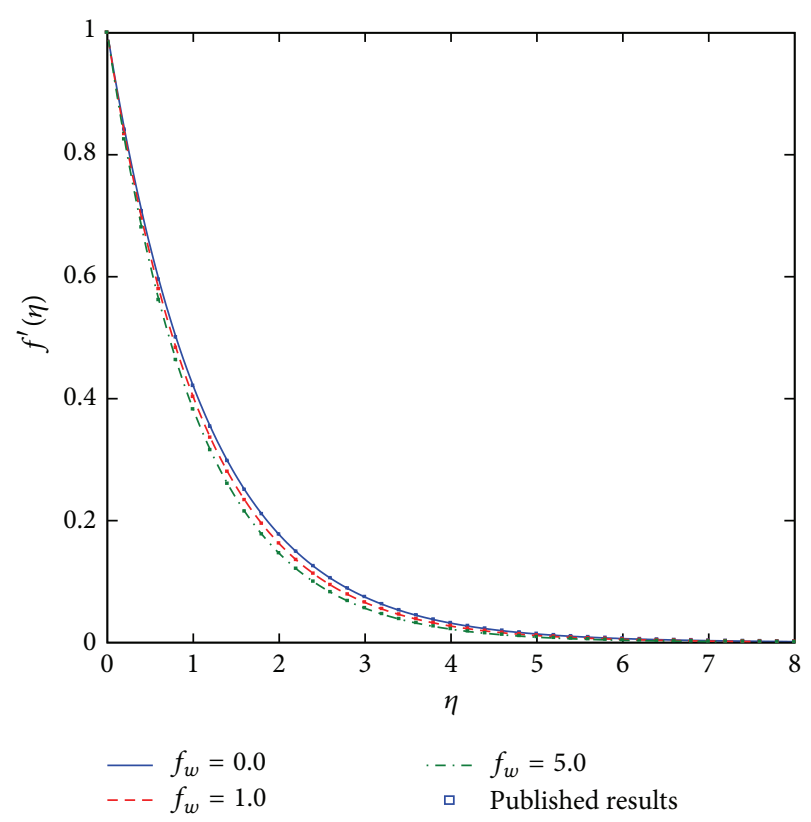

FIGURE 5: Verification of $f^{\prime}(\eta)$ obtained by the 20th order of HAM solution with pervious published paper [40] when $k_{1}=1, \mathrm{Mn}=0.5$, $\lambda=0, n=1$, and $\gamma=0.65$.

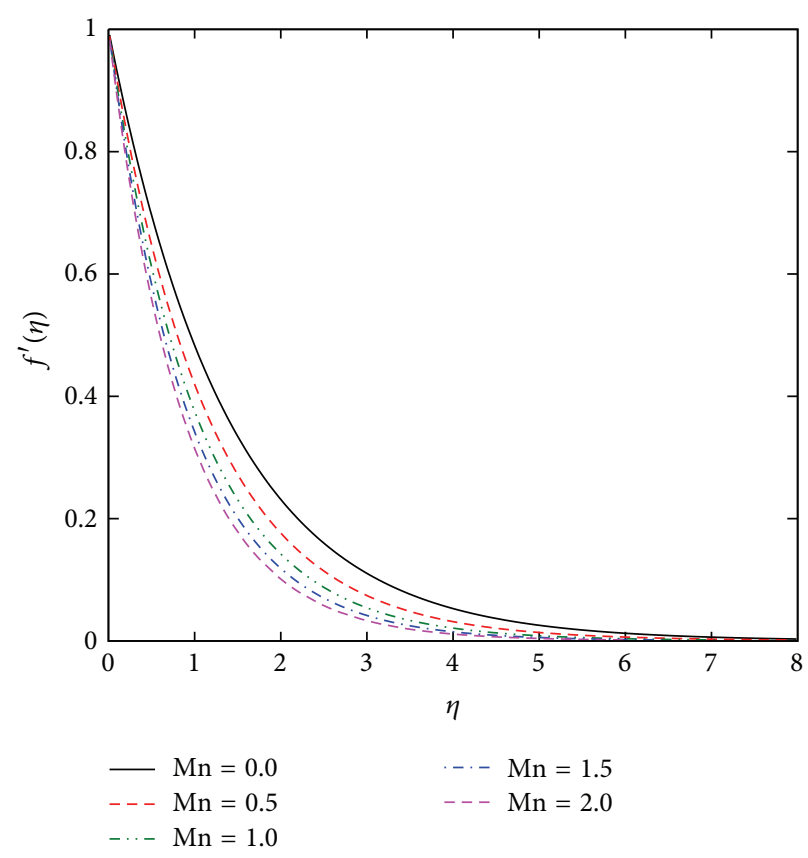

FIgURE 6: The effect of Mn on velocity profile when $k_{1}=1, \lambda=0.4$, $\operatorname{Pr}=0.71, \mathrm{Du}=0.1, \mathrm{Sr}=0.5, n=0.5, f_{w}=1.0$, and Le $=1.5$.

temperature and concentration [43]. In this paper the value of $n$ is considered to be 0.5 . The effect of magnetic parameter on the velocity is plotted in Figure 6. Transverse magnetic field parameter Mn creates a drag force, namely, Lorentz force that resists the flow and slows down the flow and causes to decrease the velocity. In Figure 7 the effect of magnetic field 


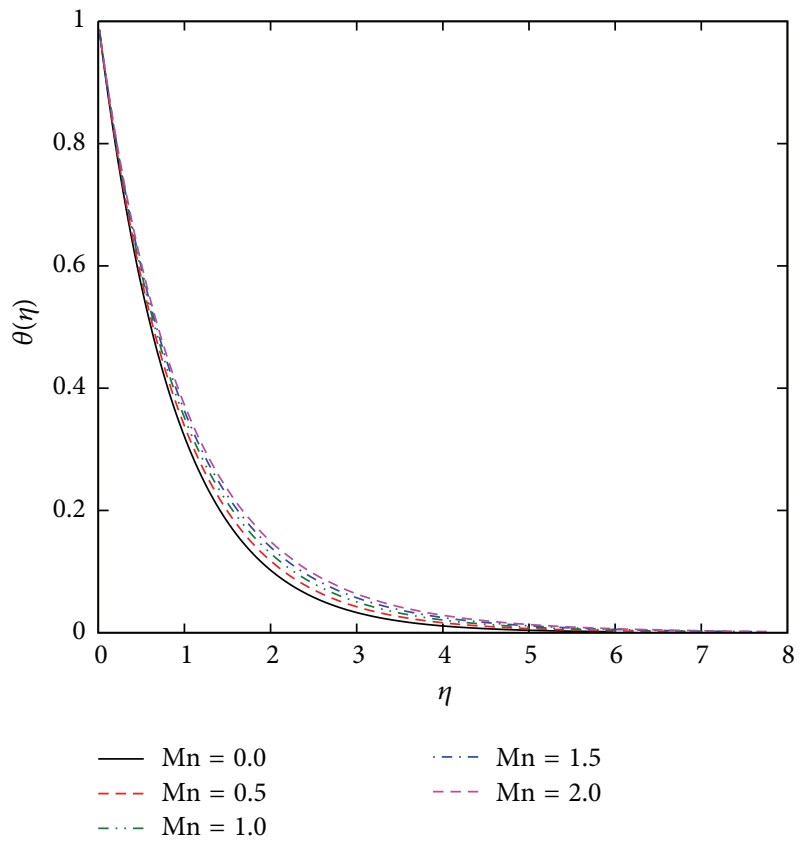

Figure 7: The effect of $\mathrm{Mn}$ on temperature profile when $k_{1}=1$, $\lambda=0.4, \operatorname{Pr}=0.71, \mathrm{Du}=0.1, \mathrm{Sr}=0.5, n=0.5, f_{w}=1.0$, and $\mathrm{Le}=1.5$.

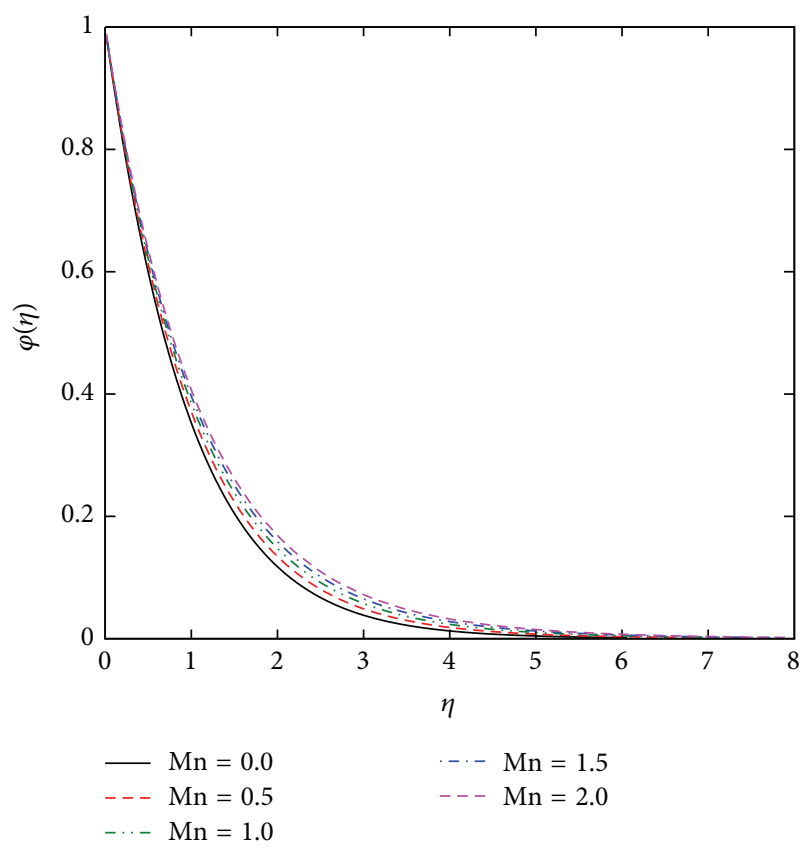

FIgURE 8: The effect of $\mathrm{Mn}$ on concentration profile when $k_{1}=1$, $\lambda=0.4, \operatorname{Pr}=0.71, \mathrm{Du}=0.1, \mathrm{Sr}=0.5, n=0.5, f_{w}=1.0$, and $\mathrm{Le}=1.5$.

parameter on temperature profiles is illustrated. Magnetic field parameter causes skin-frictional heating and so the wall temperature increases and the thickness of thermal boundary-layer increases. The effect of $\mathrm{Mn}$ is to increase the concentration profile (Figure 8). The governing equations are coupled together only with the buoyancy parameters.

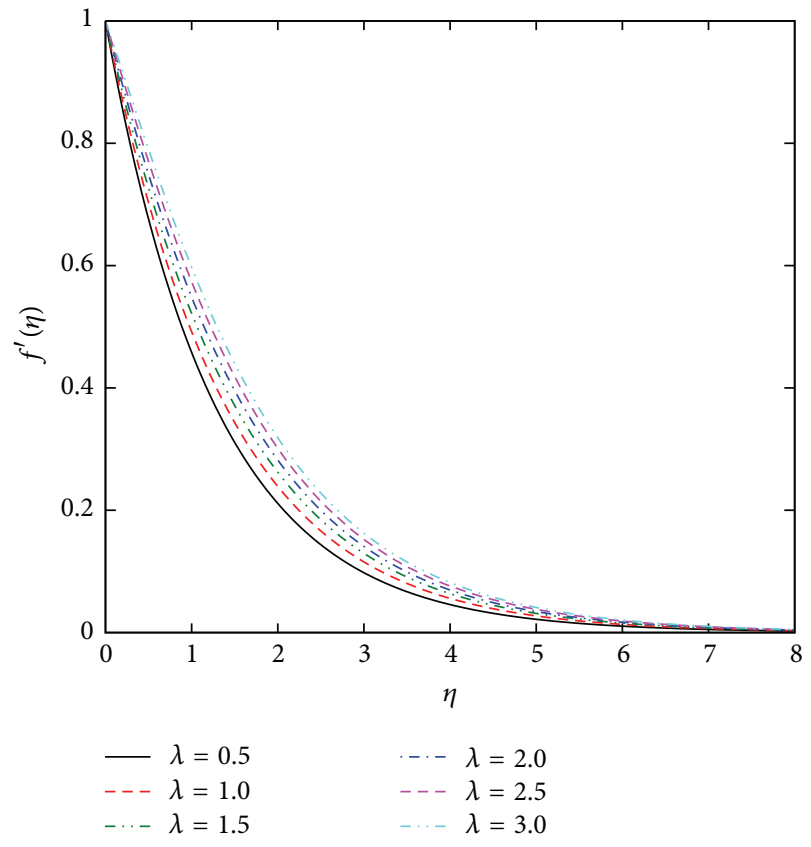

FIGURE 9: The effect of $\lambda$ on velocity profile when $k_{1}=1, \mathrm{Mn}=0.5$, $\mathrm{Pr}=0.71, \mathrm{Du}=0.1, \mathrm{Sr}=0.5, n=0.5, f_{w}=0.25$, and $\mathrm{Le}=2$.

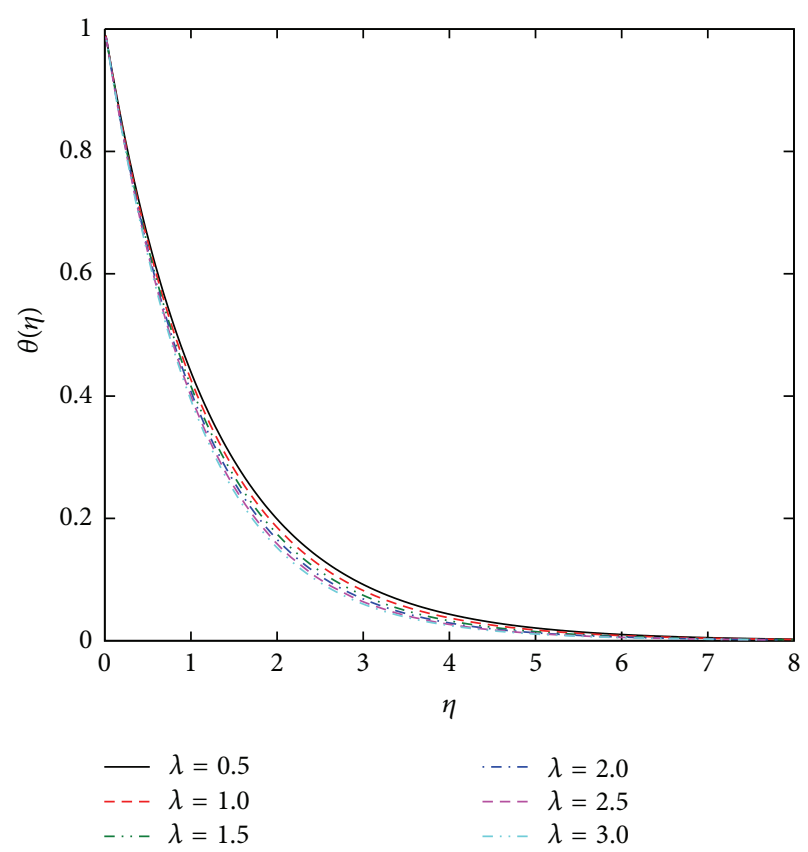

FIgURE 10: The effect of $\lambda$ on temperature profile when $k_{1}=1, \mathrm{Mn}=$ $0.5, \operatorname{Pr}=0.71, \mathrm{Du}=0.1, \mathrm{Sr}=0.5, n=0.5, f_{w}=0.25$, and Le $=2$.

When $\lambda$ increases, the Grashof number accelerates the fluid so the velocity and the boundary-layer thickness increases, as shown in Figure 9. The effect of $\lambda$ on temperature and concentration profiles is shown in Figures 10 and 11. Both the thermal and concentration boundary-layer thicknesses decrease with the increase in the value of buoyancy parameter. The 


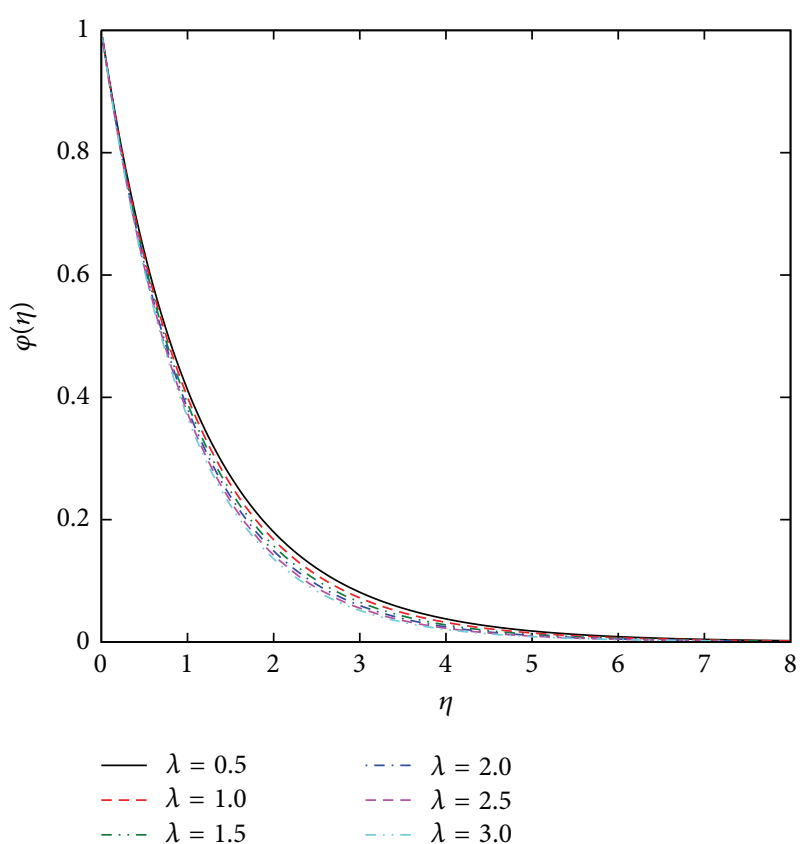

FIGURE 11: The effect of $\lambda$ on concentration profile when $k_{1}=1$, $\mathrm{Mn}=0.5, \mathrm{Pr}=0.71, \mathrm{Du}=0.1, \mathrm{Sr}=0.5, n=0.5, f_{w}=0.25$, and $\mathrm{Le}=2$.

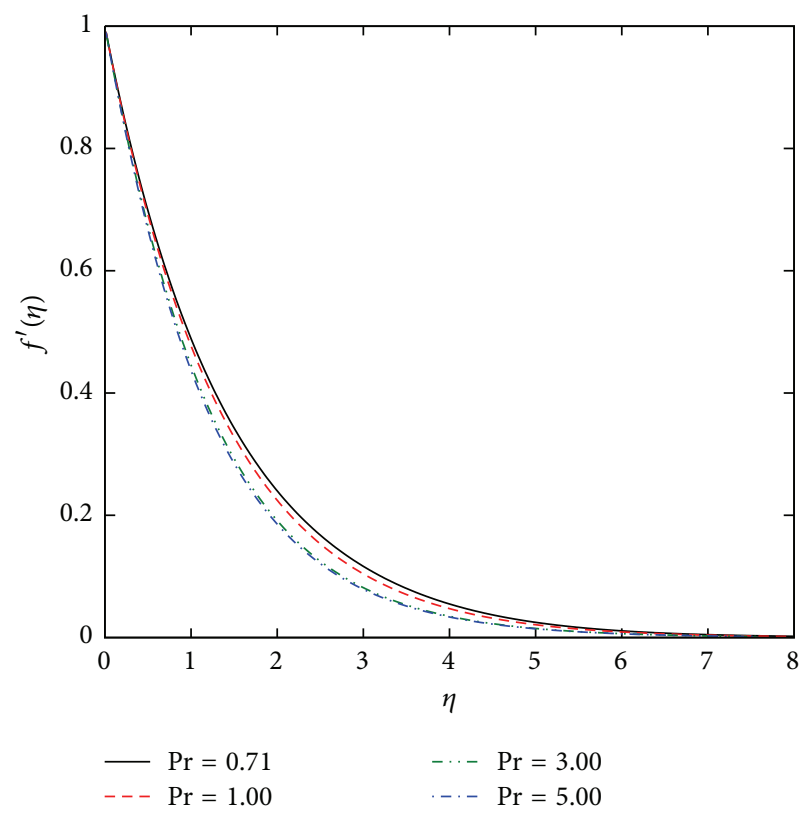

FIGURE 12: The effect of Pr on velocity profile when $k_{1}=1, \mathrm{Mn}=0.5$, $\lambda=0.6, \mathrm{Du}=0.2, \mathrm{Sr}=0.25, n=0.5, f_{w}=0.1$, and $\mathrm{Le}=2$.

effects of Prandtl number on velocity, temperature, and concentration distributions are illustrated in Figures 12-14, respectively. Increase in Pr leads to increase in kinematic viscosity and velocity decreases. It is clearly shown that with the increase in Pr the velocity profiles descends (Figure 12). With the increase in Prandtl number the thermal diffusion decreases, so the thermal boundary-layer becomes thinner

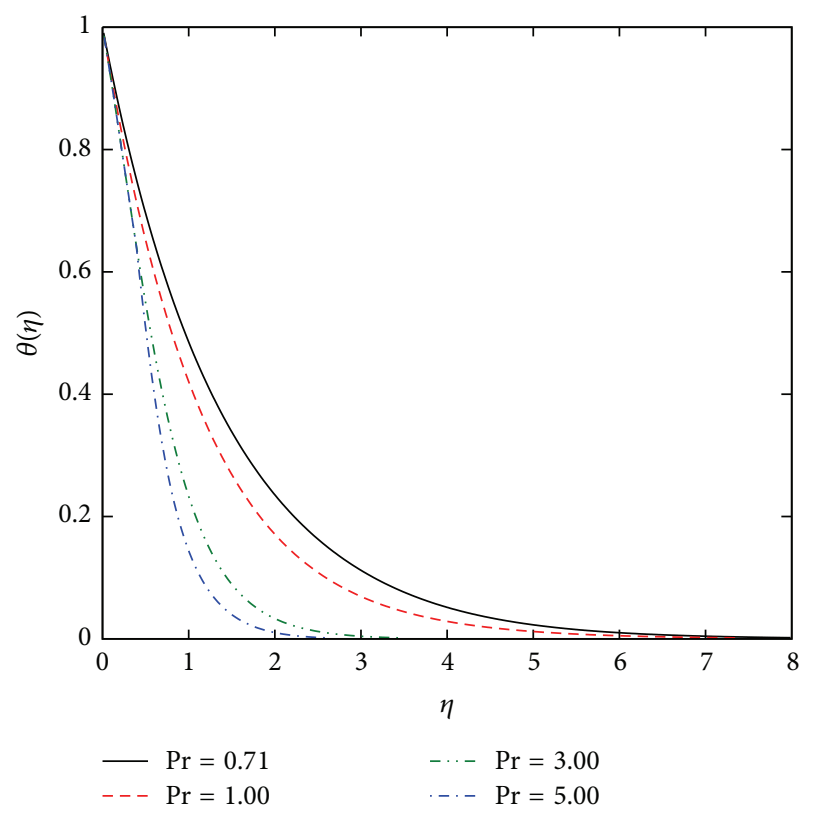

FIGURE 13: The effect of Pr on temperature profile when $k_{1}=1, \mathrm{Mn}=$ $0.5, \lambda=0.6, \mathrm{Du}=0.2, \mathrm{Sr}=0.25, n=0.5, f_{w}=0.1$, and Le $=2$.

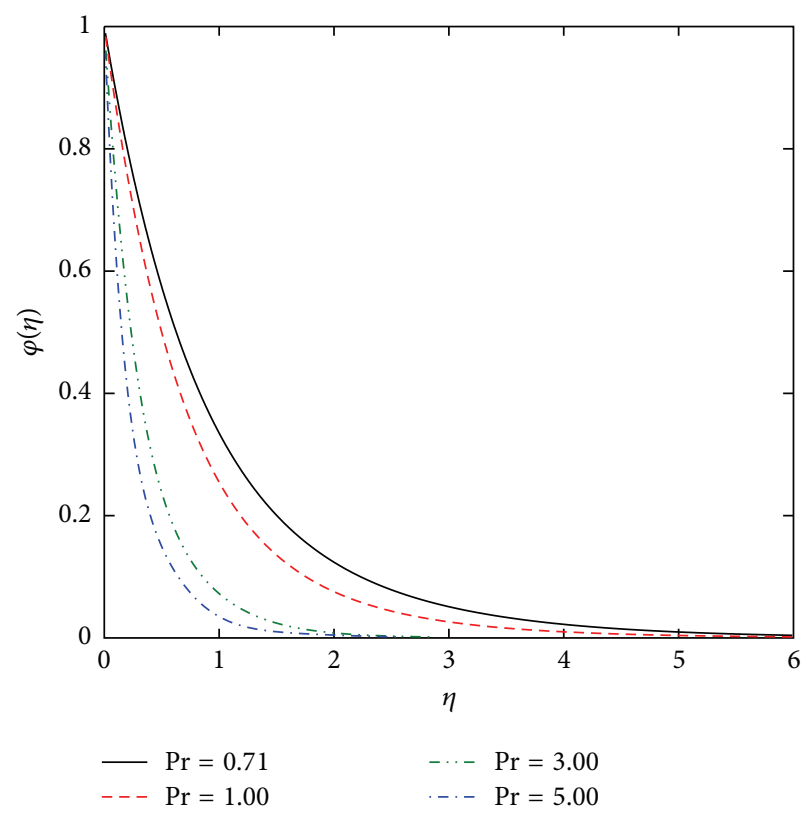

FIgURE 14: The effect of Pr on concentration profile when $k_{1}=1$, $\mathrm{Mn}=0.5, \lambda=0.6, \mathrm{Du}=0.2, \mathrm{Sr}=0.25, n=0.5, f_{w}=0.1$, and $\mathrm{Le}=2$.

and temperature decreases. A fluid with larger Pr and higher heat capacity increases the heat transfer [40] (Figure 13). The Pr reduces the concentration distribution just the same as its effect on temperature profile (Figure 14). The Soret effect is a mass flux due to a temperature gradient and the Dufour effect is enthalpy flux due to a concentration gradient and appears in the energy equation. The effects of Soret and 


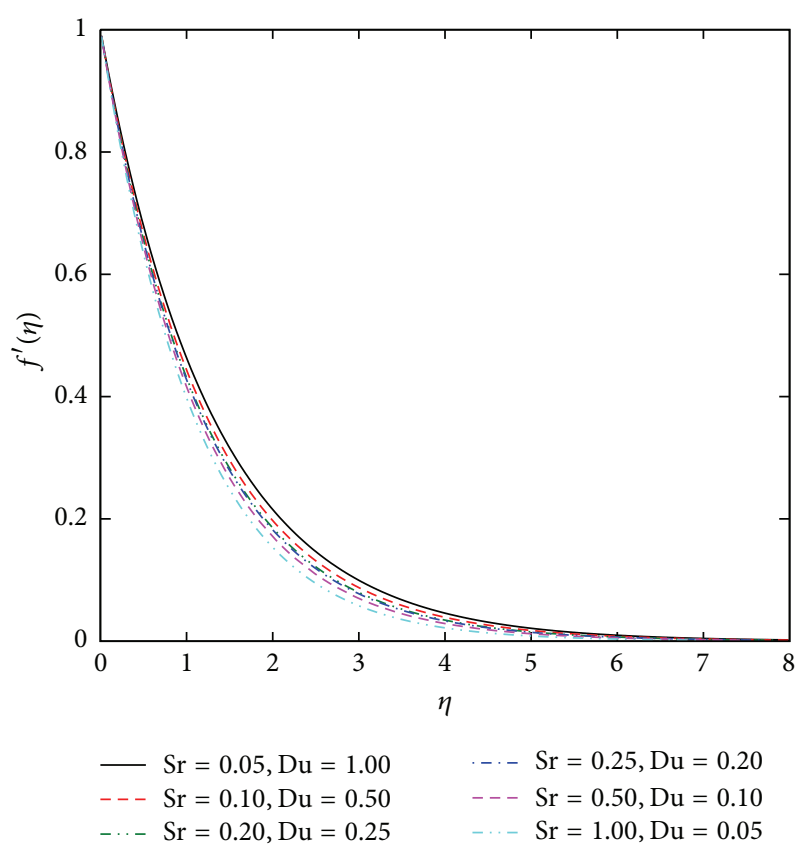

FIGURE 15: The effect of Du, Sr on velocity profile when $k_{1}=1, \mathrm{Mn}=$ $0.5, \lambda=0.6, \operatorname{Pr}=0.71, n=0.5, f_{w}=1.0$, and Le $=1$.

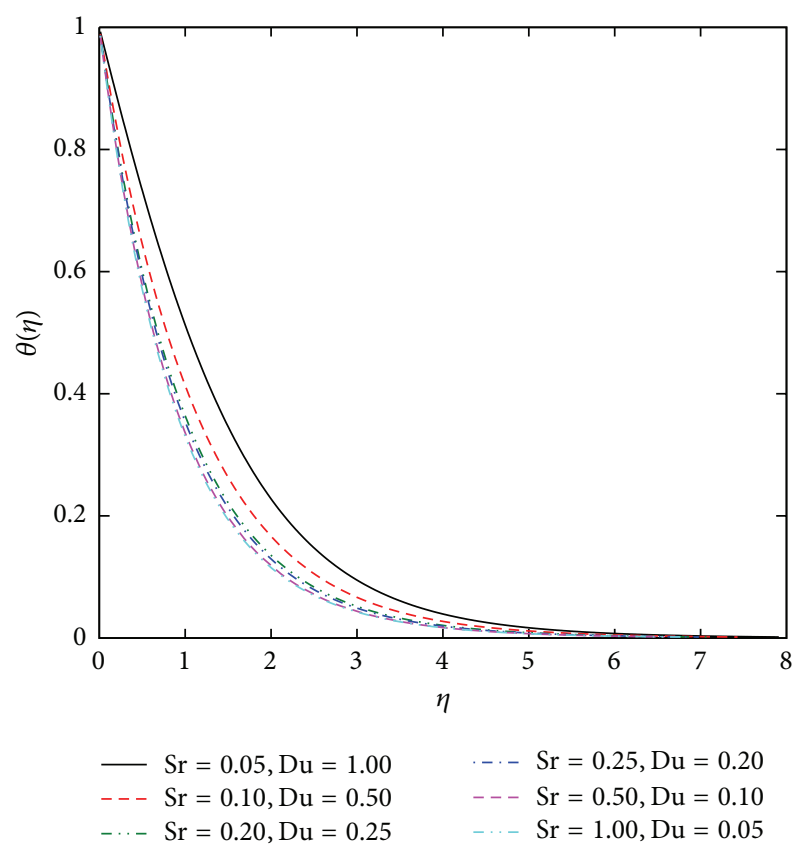

FIGURE 16: The effect of $\mathrm{Du}, \mathrm{Sr}$ on temperature profile when $k_{1}=1$, $\mathrm{Mn}=0.5, \lambda=0.6, \operatorname{Pr}=0.71, n=0.5, f_{w}=1.0$, and Le $=1$.

Dufour numbers on velocity, temperature, and concentration profiles are plotted in Figures 15, 16, and 17, respectively. We considered the effects of $\mathrm{Du}$ and $\mathrm{Sr}$ so that their product remains constant at 0.05 . As one can see the increase in the value of $\mathrm{Sr}$ or decrease in $\mathrm{Du}$ descends the velocity and temperature profiles and enhances the concentration distribution. Increase in Soret number cools the fluid and

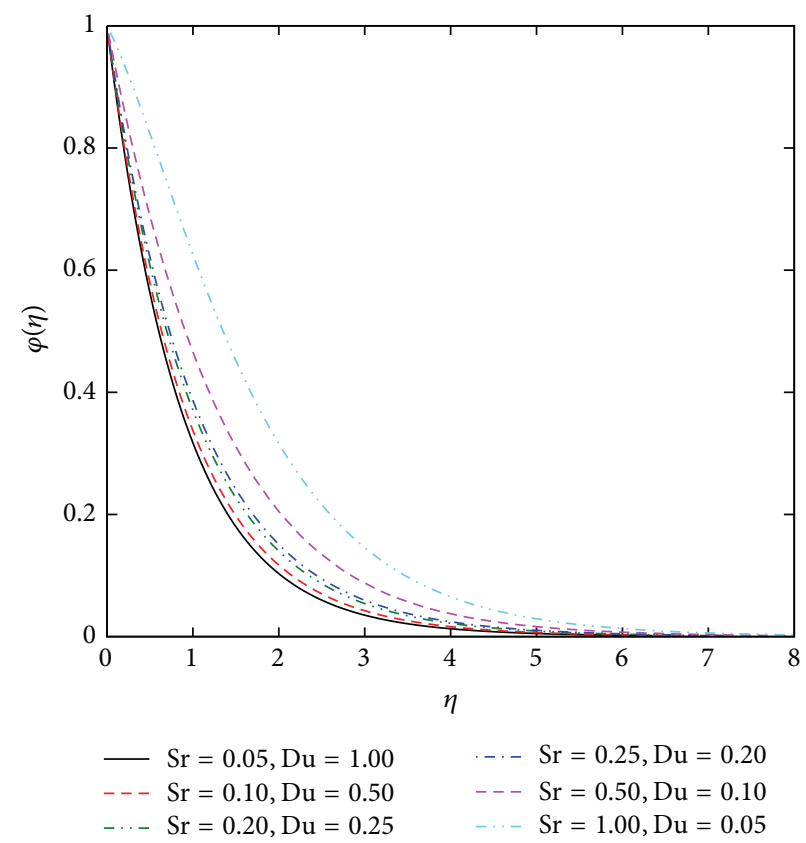

FIGURE 17: The effect of $\mathrm{Du}, \mathrm{Sr}$ on concentration profile when $k_{1}=1$, $\mathrm{Mn}=0.5, \lambda=0.6, \operatorname{Pr}=0.71, n=0.5, f_{w}=1.0$, and $\mathrm{Le}=1$.

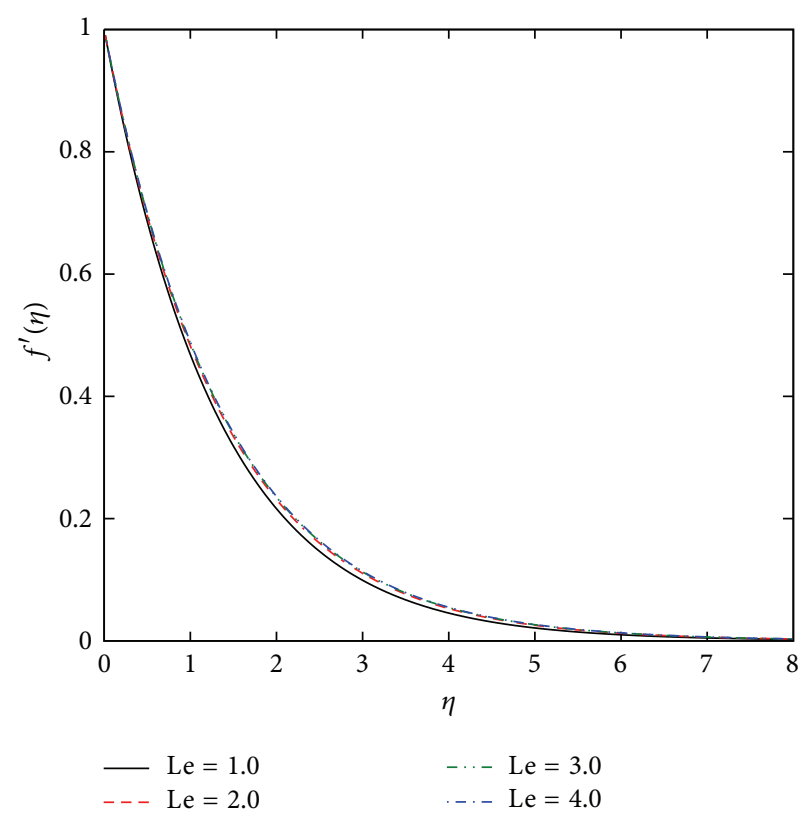

FIGURE 18: The effect of Le on velocity profile when $k_{1}=1, \mathrm{Mn}=0.2$, $\lambda=0.4, \operatorname{Pr}=0.71, \mathrm{Du}=0.1, \mathrm{Sr}=0.5, f_{w}=0.5$, and $n=0.5$.

reduces the temperature [43]. Lewis number is the ratio of thermal diffusivity to mass diffusivity. The Lewis number can also be expressed as the ratio of the Schmidt number to the Prandtl number ( $\mathrm{Le}=\mathrm{Sc} / \mathrm{Pr}$ ), where $\mathrm{Sc}=v / D_{e}$ is the Schmidt number. Figure 18 displays the effect of Lewis number on the velocity profile. The effect of increasing the value of Le on the velocity is as the same as the effect of 


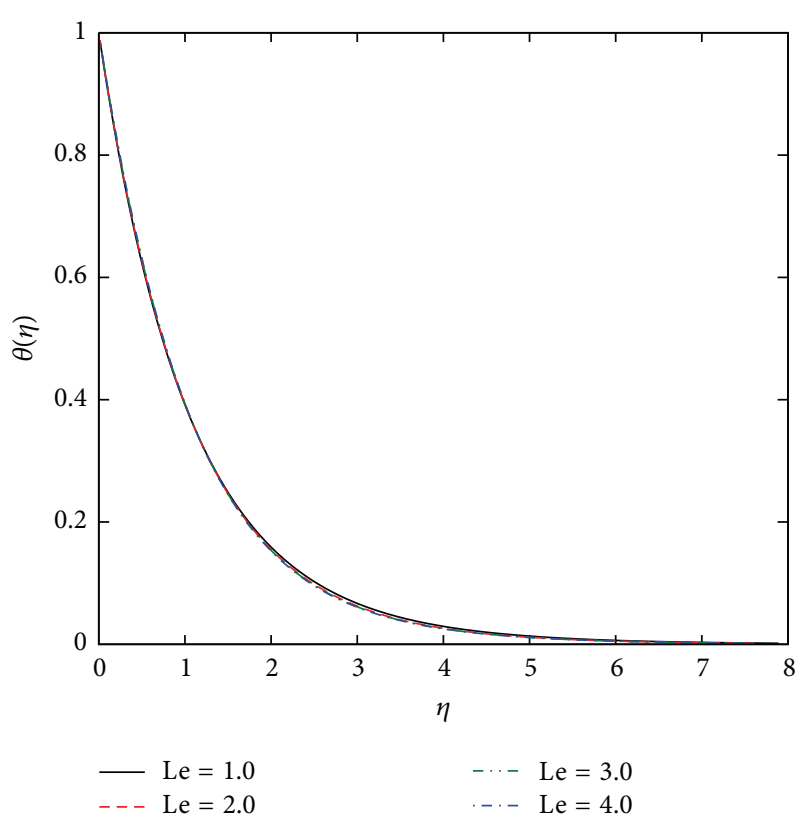

FIgURE 19: The effect of Le on temperature profile when $k_{1}=1$, $\mathrm{Mn}=0.2, \lambda=0.4, \operatorname{Pr}=0.71, \mathrm{Du}=0.1, \mathrm{Sr}=0.5, f_{w}=0.5$, and $n=0.5$.

decreasing the value of $\mathrm{Pr}$ and it can be easily understood that with the enhancement of Le the velocity distribution increases. The effect of Le on temperature profile is presented in Figure 19. The temperature decreases with the increase in Lewis number similar to the results presented by Hayat et al. [41]. With the increase in Le the mass diffusivity decreases and the concentration descends (Figure 20). It should be noticed that $n=1$ permits complete similarity solutions of the equations, where $k_{1}$ and $\lambda$ are constants and not $f(x)$. However in this problem $k_{1}$ must be constant and $n$ is selected equal to 0.5 in order to reach the local similarity solution.

\section{Conclusion}

In the present investigation, an analysis is carried out in order to study the steady magneto hydrodynamic incompressible viscoelastic fluid flow over a stretching surface in the presence of the Soret and Dufour effects analytically via HAM with two auxiliary parameters. Analytical solutions are obtained using the homotopy analysis method and its residual was reduced by using the second auxiliary parameter. These analytical solutions show excellent agreement with the data available in the literature (Figures 3-5). The effect of $\mathrm{Mn}$ is to decrease the velocity while increasing the thermal boundary-layer. The effect of increasing the buoyancy parameter is to reduce both the thermal and concentration boundary-layer thicknesses. The effect of increasing Sr or decreasing Du tends to decreases the velocity and temperature profiles while enhancing the concentration distribution. The temperature profiles are not sensitive to increasing Le however the concentration profiles are very sensitive.

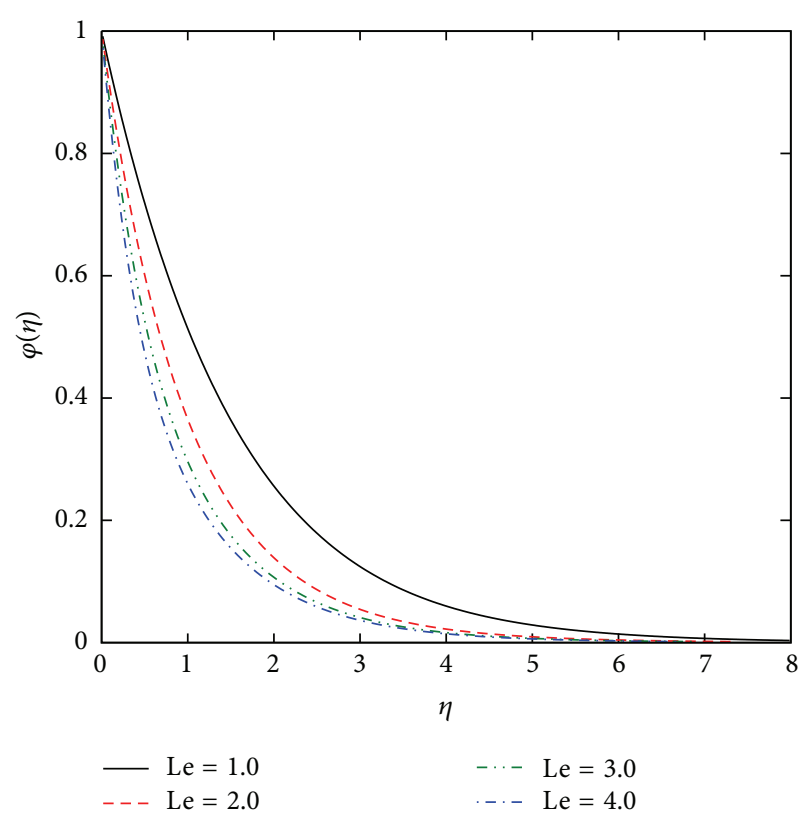

Figure 20: The effect of Le on concentration profile when $k_{1}=1$, $\mathrm{Mn}=0.2, \lambda=0.4, \mathrm{Pr}=0.71, \mathrm{Du}=0.1, \mathrm{Sr}=0.5, f_{w}=0.5$, and $n=0.5$.

\section{Nomenclature}

$a, b, c$ : Constant values [-]

$B(x)$ : Magnetic field $\left[\mathrm{kg} \mathrm{s}^{-2} \mathrm{~A}^{-1}\right]$

$c_{i}: \quad$ Arbitrary constant [-]

C: $\quad$ Concentration $\left[\mathrm{kg} \mathrm{m}^{-2}\right]$

$c_{p}$ : Specific heat at constant pressure

$\left[\mathrm{J} \mathrm{kg}^{-1} \mathrm{~K}^{-1}\right]$

$D_{e}: \quad$ Coefficient of mass diffusivity $\left[\mathrm{m}^{2} \mathrm{~s}^{-1}\right]$

Du: Dufour number $\left(=D_{e} k_{T}\left(C_{w}-C_{\infty}\right)\left(c_{s} c_{P}\left(T_{w}-T_{\infty}\right) v\right)^{-1}\right)[-]$

$\hbar$ : Auxiliary nonzero parameter

$\mathscr{H}: \quad$ Auxiliary function

$\mathscr{L}: \quad$ Auxiliary linear operator

Le: Lewis number $\left(=\alpha D_{m}^{-1}\right)[-]$

Mn: Magnetic field parameter $\left(=\sigma B_{0}^{2} a^{-1} \rho^{-1}\right)[-]$

$\mathcal{N}: \quad$ Nonlinear operator

$N$ : Constant dimensionless concentration buoyancy parameter

Pr: $\quad$ Prandtl number $\left(=v \alpha^{-1}\right)[-]$

$\mathrm{Re}_{x}: \quad$ Reynolds number $\left(=u_{w} x v^{-1}\right)[-]$

Sr: $\quad$ Soret number $\left(=D_{e} k_{T}\left(T_{w}-T_{\infty}\right)\left(T_{m} \alpha\left(C_{w}-C_{\infty}\right)\right)^{-1}\right)[-]$

$T_{m}$ : $\quad$ Mean fluid temperature [K].

\section{Greek Letters}

$\alpha$ : Thermal diffusivity $\left[\mathrm{m}^{2} \mathrm{~s}^{-1}\right]$

$\beta_{T}$ : Coefficient of thermal expansion $\left[\mathrm{K}^{-1}\right]$

$\beta_{C}$ : Coefficient of thermal expansion with concentration $\left[\mathrm{kg}^{-1} \mathrm{~m}^{3}\right]$ 
$\varphi$ : Dimensionless fluid concentration

$$
\left(=\left(C-C_{\infty}\right)\left(C_{w}-C_{\infty}\right)^{-1}\right)[-]
$$

$\gamma$ : The second auxiliary parameter

$\eta$ : Similarity variable

$$
\left(=u_{w}^{0.5} v^{-0.5} x^{-0.5} y\right)[-]
$$

$\theta$ : Dimensionless fluid temperature

$$
\left(=\left(T-T_{\infty}\right)\left(T_{w}-T_{\infty}\right)^{-1}\right)[-]
$$

$\rho$ : Density $\left[\mathrm{kg} \mathrm{m}^{-3}\right]$

$\sigma$ : Fluid electrical conductivity $\left[\mathrm{S} \mathrm{m}^{-1}\right]$

$\lambda$ : Buoyancy parameter $\left(=\mathrm{Gr}_{x} \mathrm{Re}_{x}^{-2}\right)[-]$

$v$ : Fluid kinematic viscosity $\left[\mathrm{m}^{2} \mathrm{~s}^{-1}\right]$

$\psi$ : Stream function.

\section{Subscripts}

$w$ : Wall condition

$\infty$ : Infinity condition.

\section{Superscript}

I: Differentiation with respect to $\eta$.

\section{Conflict of Interests}

All the authors have no conflict of interests to report.

\section{Acknowledgments}

The authors express their gratitude to the anonymous referees for their constructive reviews of the paper and for helpful comments. The authors extend their appreciation to the Deanship of Scientific Research at King Saud University for funding this work through the research group Project no. RGP-VPP-080.

\section{References}

[1] S. Abel, K. V. Prasad, and A. Mahaboob, "Buoyancy force and thermal radiation effects in MHD boundary layer visco-elastic fluid flow over continuously moving stretching surface," International Journal of Thermal Sciences, vol. 44, no. 5, pp. 465-476, 2005.

[2] R. Tamizharasi and V. Kumaran, "Pressure in MHD/Brinkman flow past a stretching sheet," Communications in Nonlinear Science and Numerical Simulation, vol. 16, no. 12, pp. 4671-4681, 2011.

[3] T. Hayat and F. A. Hendi, "Thermal-diffusion and diffusionthermo effects on MHD three-dimensional axisymmetric flow with Hall andion-slip currents," Journal of American Science, vol. 8, pp. 284-294, 2012.

[4] S. P. A. Devi and R. U. Devi, "Soret and Dufour effects on MHD slip flow with thermal radiation over a porous rotating infinite disk," Communications in Nonlinear Science and Numerical Simulation, vol. 16, no. 4, pp. 1917-1930, 2011.

[5] A. A. Afify, "Similarity solution in MHD: effects of thermal diffusion and diffusion thermo on free convective heat and mass transfer over a stretching surface considering suction or injection," Communications in Nonlinear Science and Numerical Simulation, vol. 14, no. 5, pp. 2202-2214, 2009.
[6] M. A. A. Hamad, M. J. Uddin, and A. I. M. Ismail, "Investigation of combined heat and mass transfer by Lie group analysis with variable diffusivity taking into account hydrodynamic slip and thermal convective boundary conditions," International Journal of Heat and Mass Transfer, vol. 55, no. 4, pp. 1355-1362, 2012.

[7] M. Ali and F. Al-Yousef, "Laminar mixed convection from a continuously moving vertical surface with suction or injection," Heat and Mass Transfer, vol. 33, no. 4, pp. 301-306, 1998.

[8] M. Ali and F. Al-Yousef, "Laminar mixed convection boundary layers induced by a linearly stretching permeable surface," International Journal of Heat and Mass Transfer, vol. 45, no. 21, pp. 4241-4250, 2002.

[9] M. E. Ali, "The effect of variable viscosity on mixed convection heat transfer along a vertical moving surface," International Journal of Thermal Sciences, vol. 45, no. 1, pp. 60-69, 2006.

[10] S. S. Das, A. Satapathy, J. K. Das, and J. P. Panda, "Mass transfer effects on MHD flow and heat transfer past a vertical porous plate through a porous medium under oscillatory suction and heat source," International Journal of Heat and Mass Transfer, vol. 52, no. 25-26, pp. 5962-5969, 2009.

[11] C.-H. Chen, "Combined heat and mass transfer in MHD free convection from a vertical surface with Ohmic heating and viscous dissipation," International Journal of Engineering Science, vol. 42, no. 7, pp. 699-713, 2004.

[12] N. F. M. Noor, S. Abbasbandy, and I. Hashim, "Heat and mass transfer of thermophoretic MHD flow over an inclined radiate isothermal permeable surface in the presence of heat source/ sink," International Journal of Heat and Mass Transfer, vol. 55, no. 7-8, pp. 2122-2128, 2012.

[13] C. R. A. Abreu, M. F. Alfradique, and A. S. Telles, "Boundary layer flows with dufour and soret effects: I: forced and natural convection," Chemical Engineering Science, vol. 61, no. 13, pp. 4282-4289, 2006.

[14] D. Pal and B. Talukdar, "Influence of fluctuating thermal and mass diffusion on unsteady MHD buoyancy-driven convection past a vertical surface with chemical reaction and Soret effects," Communications in Nonlinear Science and Numerical Simulation, vol. 17, no. 4, pp. 1597-1614, 2012.

[15] J. A. Gbadeyan, A. S. Idowu, A. W. Ogunsola, O. O. Agboola, and P. O. Olanrewaju, "Heat and mass transfer for Soret and Dufours effect on mixed convection boundary layer flow over a stretching vertical surface in a porous medium filled with a viscoelastic fluid in the presence of magnetic field," Global Journal of Science Frontier Research, vol. 11, pp. 97-114, 2011.

[16] V. R. Prasad, B. Vasu, O. A. Bég, and R. D. Parshad, "Thermal radiation effects on magnetohydrodynamic free convection heat and mass transfer from a sphere in a variable porosity regime," Communications in Nonlinear Science and Numerical Simulation, vol. 17, no. 2, pp. 654-671, 2012.

[17] D. Pal and H. Mondal, "MHD non-Darcian mixed convection heat and mass transfer over a non-linear stretching sheet with Soret-Dufour effects and chemical reaction," International Communications in Heat and Mass Transfer, vol. 38, no. 4, pp. 463-467, 2011.

[18] D. Pal and H. Mondal, "Effects of Soret Dufour, chemical reaction and thermal radiation on MHD non-Darcy unsteady mixed convective heat and mass transfer over a stretching sheet," Communications in Nonlinear Science and Numerical Simulation, vol. 16, no. 4, pp. 1942-1958, 2011.

[19] D. Pal and H. Mondal, "MHD non-Darcy mixed convective diffusion of species over a stretching sheet embedded in a porous medium with non-uniform heat source/sink, variable viscosity and Soret effect," Communications in Nonlinear Science and Numerical Simulation, vol. 17, no. 2, pp. 672-684, 2012. 
[20] M. A. Mansour, N. F. El-Anssary, and A. M. Aly, "Effects of chemical reaction and thermal stratification on MHD free convective heat and mass transfer over a vertical stretching surface embedded in a porous media considering Soret and Dufour numbers," Chemical Engineering Journal, vol. 145, no. 2, pp. 340-345, 2008.

[21] O. A. Bég, V. R. Prasad, B. Vasu, N. B. Reddy, Q. Li, and R. Bhargava, "Free convection heat and mass transfer from an isothermal sphere to a micropolar regime with Soret/Dufour effects," International Journal of Heat and Mass Transfer, vol. 54, no. 1-3, pp. 9-18, 2011.

[22] M. S. Alam, M. M. Rahman, and M. A. Sattar, "Effects of variable suction and thermophoresis on steady MHD combined freeforced convective heat and mass transfer flow over a semiinfinite permeable inclined plate in the presence of thermal radiation," International Journal of Thermal Sciences, vol. 47, no. 6, pp. 758-765, 2008.

[23] B.-C. Tai and M.-I. Char, "Soret and Dufour effects on free convection flow of non-Newtonian fluids along a vertical plate embedded in a porous medium with thermal radiation," International Communications in Heat and Mass Transfer, vol. 37, no. 5, pp. 480-483, 2010.

[24] A. Mahdy, "MHD non-Darcian free convection from a vertical wavy surface embedded in porous media in the presence of Soret and Dufour effect," International Communications in Heat and Mass Transfer, vol. 36, no. 10, pp. 1067-1074, 2009.

[25] A. Mahdy, "Soret and Dufour effect on double diffusion mixed convection from a vertical surface in a porous medium saturated with a non-Newtonian fluid," Journal of Non-Newtonian Fluid Mechanics, vol. 165, no. 11-12, pp. 568-575, 2010.

[26] D. Pal and S. C. Sewli, "Mixed convection magnetohydrodynamic heat and mass transfer past a stretching surface in a micropolar fluid-saturated porous medium under the influence of Ohmic heating, Soret and Dufour effects," Communications in Nonlinear Science and Numerical Simulation, vol. 16, no. 3, pp. 1329-1346, 2011.

[27] R. Tsai and J. S. Huang, "Numerical study of Soret and Dufour effects on heat and mass transfer from natural convection flow over a vertical porous medium with variable wall heat fluxes," Computational Materials Science, vol. 47, no. 1, pp. 23-30, 2009.

[28] S. Liao, Beyond Perturbation: Introduction to the Homotopy Analysis Method, Chapman \& Hall/CRC Press, 2004.

[29] S. Liao, "On the homotopy analysis method for nonlinear problems," Applied Mathematics and Computation, vol. 147, no. 2, pp. 499-513, 2004.

[30] M. M. Rashidi, T. Hayat, E. Erfani, S. A. M. Pour, and A. A. Hendi, "Simultaneous effects of partial slip and thermal-diffusion and diffusion-thermo on steady MHD convective flow due to a rotating disk," Communications in Nonlinear Science and Numerical Simulation, vol. 16, no. 11, pp. 4303-4317, 2011.

[31] M. Mustafa, T. Hayat, I. Pop, S. Asghar, and S. Obaidat, "Stagnation-point flow of a nanofluid towards a stretching sheet," International Journal of Heat and Mass Transfer, vol. 54, no. 2526, pp. 5588-5594, 2011.

[32] M. M. Rashidi and S. A. M. Pour, "Analytic approximate solutions for unsteady boundary-layer flow and heat transfer due to a stretching sheet by homotopy analysis method," Nonlinear Analysis: Modelling and Control, vol. 15, no. 1, pp. 83-95, 2010.

[33] Z. Abbas, Y. Wang, T. Hayat, and M. Oberlack, "Mixed convection in the stagnation-point flow of a Maxwell fluid towards a vertical stretching surface," Nonlinear Analysis: Real World Applications, vol. 11, no. 4, pp. 3218-3228, 2010.
[34] S. Dinarvand, A. Doosthoseini, E. Doosthoseini, and M. M. Rashidi, "Series solutions for unsteady laminar MHD flow near forward stagnation point of an impulsively rotating and translating sphere in presence of buoyancy forces," Nonlinear Analysis: Real World Applications, vol. 11, no. 2, pp. 1159-1169, 2010.

[35] T. Hayat, M. Nawaz, S. Asghar, and S. Mesloub, “Thermal-diffusion and diffusion-thermo effects on axisymmetric flow of a second grade fluid," International Journal of Heat and Mass Transfer, vol. 54, no. 13-14, pp. 3031-3041, 2011.

[36] Z. Ziabakhsh, G. Domairry, and H. R. Ghazizadeh, "Analytical solution of the stagnation-point flow in a porous medium by using the homotopy analysis method," Journal of the Taiwan Institute of Chemical Engineers, vol. 40, no. 1, pp. 91-97, 2009.

[37] P. D. Weidman and M. E. Ali, "Aligned and nonaligned radial stagnation flow on a stretching cylinder," European Journal of Mechanics, B/Fluids, vol. 30, no. 1, pp. 120-128, 2011.

[38] M. M. Rashidi, M. Ashraf, B. Rostami, M. T. Rastegari, and S. Bashir, "Mixed convection boundary-layer flow of a micro polar fluid towards a heated shrinking sheet by homotopy analysis method," Thermal Science, 2013.

[39] M. M. Rashidi, E. Momoniat, and B. Rostami, "Analytic approximate solutions for MHD boundary-layer viscoelastic fluid flow over continuously moving stretching surface by homotopy analysis method with two auxiliary parameters," Journal of Applied Mathematics, vol. 2012, Article ID 780415, 19 pages, 2012.

[40] C.-H. Chen, "On the analytic solution of MHD flow and heat transfer for two types of viscoelastic fluid over a stretching sheet with energy dissipation, internal heat source and thermal radiation," International Journal of Heat and Mass Transfer, vol. 53, no. 19-20, pp. 4264-4273, 2010.

[41] T. Hayat, M. Mustafa, and I. Pop, "Heat and mass transfer for Soret and Dufour's effect on mixed convection boundary layer flow over a stretching vertical surface in a porous medium filled with a viscoelastic fluid," Communications in Nonlinear Science and Numerical Simulation, vol. 15, no. 5, pp. 1183-1196, 2010.

[42] A. Ishak, R. Nazar, and I. Pop, "MHD mixed convection boundary layer flow towards a stretching vertical surface with constant wall temperature," International Journal of Heat and Mass Transfer, vol. 53, no. 23-24, pp. 5330-5334, 2010.

[43] O. A. Bég, A. Bakier, R. Prasad, and S. K. Ghosh, "Numerical modelling of non-similar mixed convection heat and species transfer along an inclined solar energy collector surface with cross diffusion effects," World Journal of Mechanics, vol. 1, pp. 185-196, 2011. 


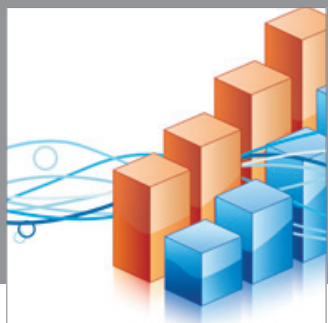

Advances in

Operations Research

mansans

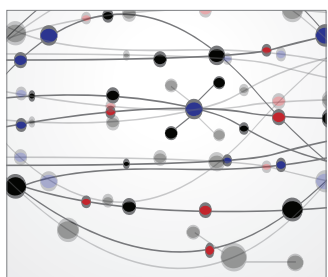

The Scientific World Journal
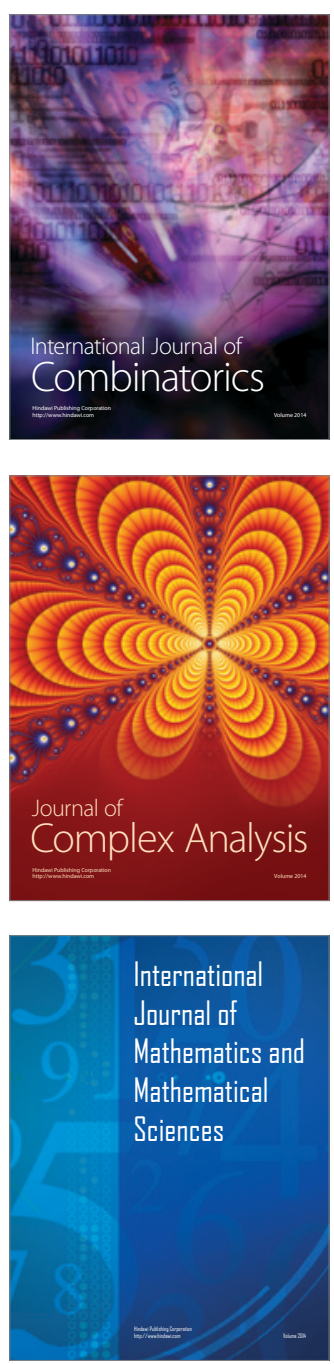
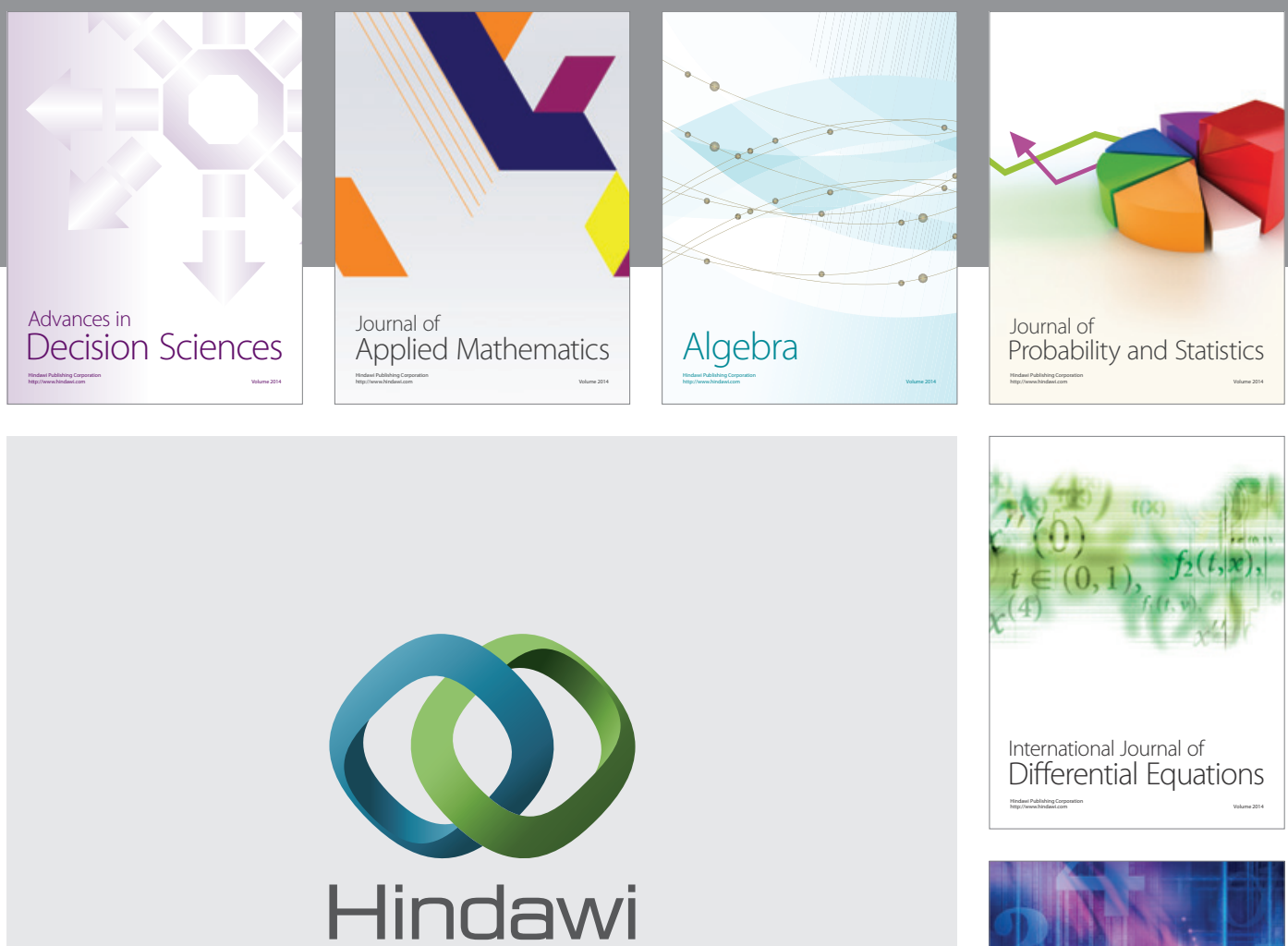

Submit your manuscripts at http://www.hindawi.com
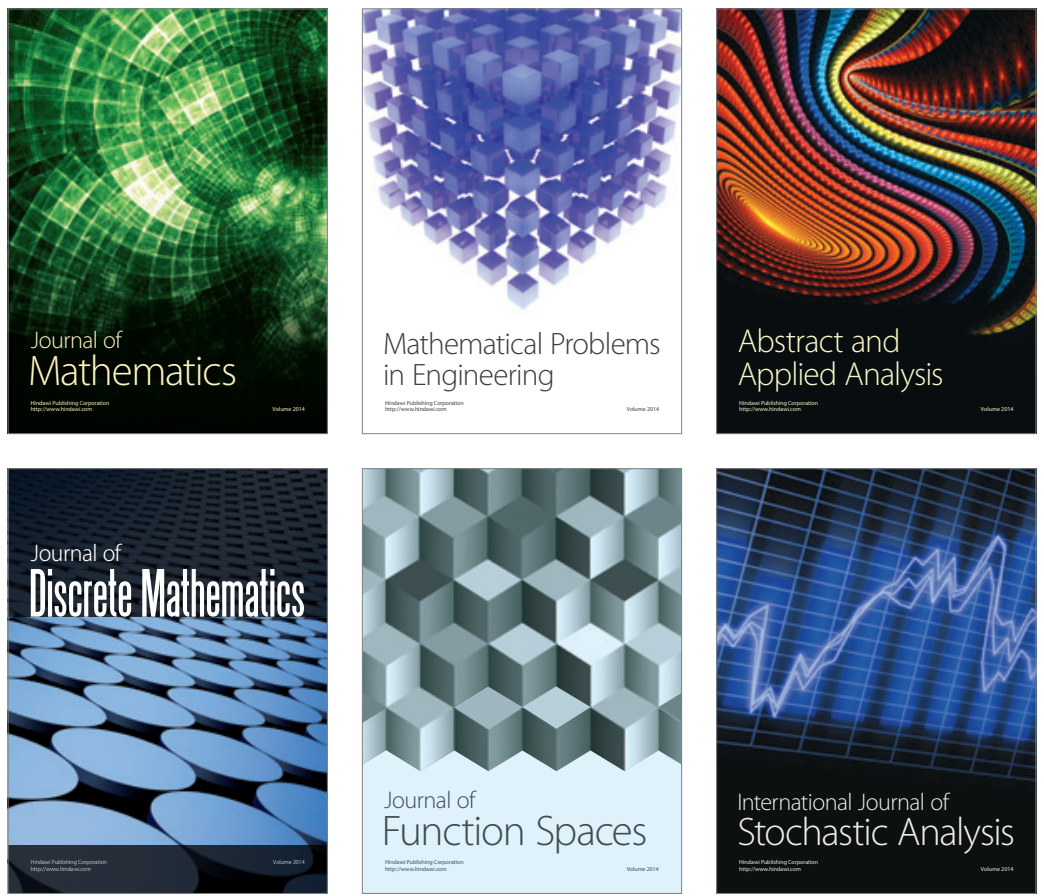

Journal of

Function Spaces

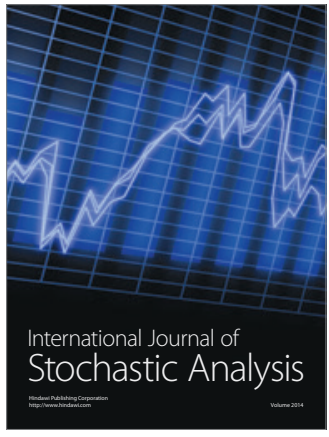

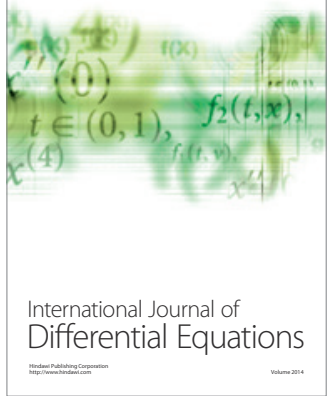
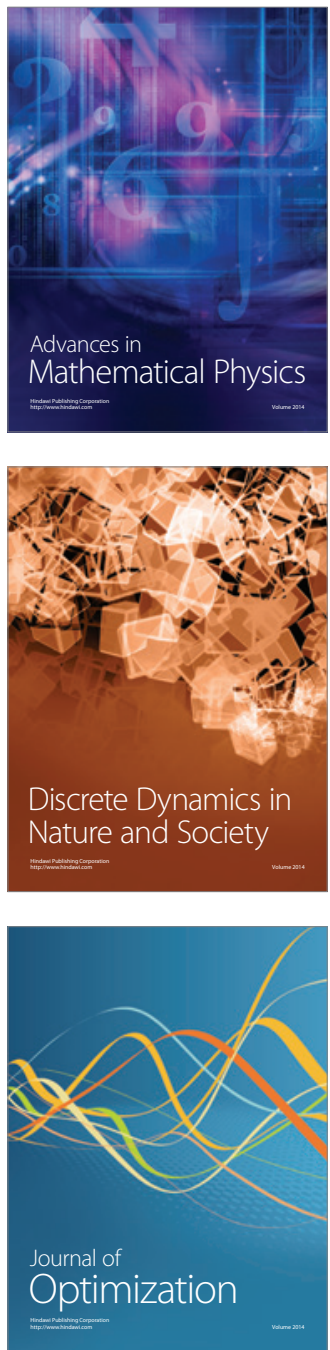